\title{
Dupuytren's Contracture, When Not Everything is Surgery
}

\author{
Manuel Robustillo*, Guillermo Dávila, Alberto Millán, Daniel Grandes, Jorge Morro and Marcos Martín Diaz \\ Plastic and Reconstructive Surgery Department, Spain
}

Received: 啙December 28, 2018; Published: 眥 January 16, 2019

*Corresponding author: Manuel Robustillo, Plastic and Reconstructive Surgery Department, Madrid, Spain

Abbreviations: MCP: Metacarpophalangeal; PIP: Proximal Interphalangeal; CCH: Collagenase Clostridium Histolyticum

\section{Introduction}

Dupuytren contracture is a common connective tissue disorder that can affect and interfere with daily activities. Palmar nodules can progress to form cords and extend into fingers causing permanent flexion of the same [1-3]. Metacarpophalangeal (MCP) joint and proximal interphalangeal (PIP) joint may be affected. Traditionally, four goals of Dupuytren's disease treatment have been proposed [4]: to correct deformity, avoid complications, shorten postoperative recovery and prevent recurrences. Surgery follow by hand recovery therapy used to be standard treatment. The risk of recurrence is a critical value in the evaluation of any treatments. Recurrence rates differ from 12 to $73 \%$ for fasciectomy/aponeurotomy and from $33 \%$ to $100 \%$ for open or needle fasciotomy/aponeurotomy depending on the series [5]. Collagenase clostridium histolyticum (CCH) has been approved as a pharmacological option for treating Dupuytren contracture with a palpable cord. About $47 \%$ of recurrence have been reported in the literature with low risk of complication [6]. We report our results from 2 years' experience with $\mathrm{CCH}$.

\section{Material and Methods}

\section{Study Design}

This 2-year prospective, open label, study was conducted from November 2016 through November 2018, in the city of Madrid, Spain. The study protocol was approved by local ethics committees. All participants provided written informed consent before the initiation of treatment and were free to leave at any time of followup.

\section{Patients}

Eligible patients were men or women 18 years old or older with Dupuytren disease who had flexion contractures of 10-100 in MCF and/or PIP finger joints that were caused by palpable cords. Patients excluded were those who were pregnant or in breastfeeding period; those with coagulation disorder or in treatment with anticoagulants (except aspirin $<150 \mathrm{mg} /$ day); received treatment with tetracycline and fluoroquinolones, neuromuscular disorders or allergy to any of the components of collagenase. The study enrolled 62 Patients with 102 Joints treated. 48 were men and 14 women. The average age at the time of treatment was 62 years. Sixty-one patients complete the study, only one was lost to follow-up because died for another cause without relation with this treatment. Joints treated included MCP and PIP, 64 and 38 respectively. 52 suffered Dupuytren disease in the right hand and 12 in the left hand. In relation to the affected finger, $6 \mathrm{had}$ contracture in flexion in the third finger, 30 in the fourth and 40 in the fifth. Of the total of patients, ten had contralateral hand surgery of Dupuytren disease (standard fasciectomy).

\section{Treatment and Study Visits}

The joints to be treated were identified at the screening visit. On day one, we realized the injection of $0.58 \mathrm{mg}$ of collagenase per palpable cord. Within each hand, the affected finger in the most ulnar aspect of the hand was injected first, within each finger the affected joint in the most proximal aspect of the finger, was injected first. We made an average of 1.02 injections per joint. Later the patient was cited in 7 days for the revision and traction of the treated finger under local anesthesia. Finally, the measurement of the degree of contracture in each joint was made on day 1 and day 30 and the patient filled out a satisfaction survey, whose value is reflected in percent (\%).

\section{Results}

In terms of efficacy, considering for it, the degrees of basal contraction in flexion, with respect to the evaluation on day 30 . We have that in the case of MCF and PIP joints, we start from a value of 
$30,16^{\circ}$ and $52,37^{\circ}$ respectively, resulting in a residual contraction of $1.41^{\circ}$ in MCF joints, and $5^{\circ}$ in PIP joints. In general, we have as a result that on average we started with a flexion contracture of $38^{\circ}$ and improving up to 2,74 ․ To be able to consider the application of collagenase, as successful, we define that the degree of residual contraction must be less than 5 degrees. In the studied sample, we obtained that $81 \%$ of the MCF joints $(52 / 64)$ and $53 \%$ of the PIP joints (20/38) could be considered as successful. Having a global success rate of $70 \%$. Regarding patient satisfaction, we have that of 61 patients surveyed, 95\% reported feeling satisfied or very satisfied. Also $91 \%$ referred a complete or almost complete correction of their functional limitation. At the time of making a subjective comparison of patients who had been previously treated with surgery in the contralateral hand (10 patients), we obtained that $80 \%$ preferred the treatment with collagenase over standard fasciectomy. Of which $80 \%$ considered it more effective, $90 \%$ less painful and $100 \%$ faster.

\section{Discussion}

Advanced Dupuytren's disease can considerably affect hand function, difficulting several activities of daily living such as shaving, tooth brushing or using the mobile phone among others. Although open fasciectomy is still considered the gold standard of Dupuytren's disease treatment, it is not free of complications such as nerve and digital artery injury, tendon rupture, infection, loss of grip strength or skin necrosis [7-8]. In this context, collagenase clostridium histolyticum is emerging as an effective alternative to surgery in patients with mild and severe Dupuytren's contracture. This procedure has been proved to have lower rates of severe complications than open fasciectomy while maintaining a similar risk of tendon rupture [9]. Furthermore, recovery from this procedure is faster than from the standard surgical treatment and there is no need for intensive hand therapy [10]. This study concludes that injectable collagenase clostridium histolyticum is an effective treatment for Dupuytren's disease, achieving a good deformity correction and hand function recovery. Although mild adverse events of injectable collagenase clostridium histolyticum such as pain, swelling or bruising are common findings in the first week after the procedure, no tendon rupture, nerve injury or grip strength loss have been reported in this study. Moreover, as described in previous studies [10], our findings support that early intervention of metacarpophalangeal and proximal interphalangeal joints may lead to a better functional result.

\section{References}

1. Rayan GM (2007) Dupuytren's disease: anatomy, pathology, presentation and treatment. J Bone Joint Surg Am 89(1): 189-198.

2. Bayat A, Cunliffe EJ, McGrouther DA (2007) Assessment of clinical severity in Dupuytren's disease. Br J Hosp Med 68(11): 604-609.

3. DiBenedetti DB, Nguyen D, Zografos L, Ziemiecki R, Zhou X (2011) Prevalence, incidence, and treatments of Dupuytren's disease in the United States: results from a population-based study. Hand (NY). 6(2): 149-158.

4. Tubiana R (1999) Surgical treatment. In: Tubiana R (Eds.). The Hand. Philadelphia: WB Saunders pp. 451-483.

5. Werker PMN, Pess GM, Van Rijssen AL, Denkler K (2012) Correction of contracture and recurrence rates of Dupuytren contracture following invasive treatment: the importance of clear definitions. J Hand Surg Am. 37(10): 2095-2105.

6. Clayton A. Peimer, Philip Blazar, Coleman, Kaplan FT, Smith T, et al. (2015) Dupuytren Contracture Recurrence following treatment with collagenase clostridium histolyticum (CORDLESS) J Hand Srg AM 40(8): 1597-1605

7. Coert JH, Nérin JP, Meek MF (2006) Results of partial fasciectomy for Dupuytren disease in 261 consecutive patients. Ann Plast Surg 57(1): 13-7.

8. Bulstrode NW, Jemec B, Smith PJ (2005) The complications of Dupuytren's contracture surgery. J Hand Surg Am 30(5): 1021-1025.

9. Peimer CA, Wilbrand S, Gerber RA, Chapman D, Szezypa PP (2015) Safety and tolerability of collagenase clostridium histolyticum and fasciectomy for Dupuytren's contracture. J Hand Surg Eur 40(2): 141-149.

10. Lawrence C Hurst MD, Badalamente MA, Hentz VR, Hotchkiss RN, M Smith, et al. (2009) Injectable collagenase clostridium histolyticum for Dupuytren's contracture. NEJM 361: 968-979.

\section{ISSN: 2574-1241}

DOI: 10.26717/BJSTR.2019.13.002365

Manuel Robustillo. Biomed J Sci \& Tech Res

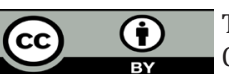

This work is licensed under Creative

Commons Attribution 4.0 License

Submission Link: https://biomedres.us/submit-manuscript.php

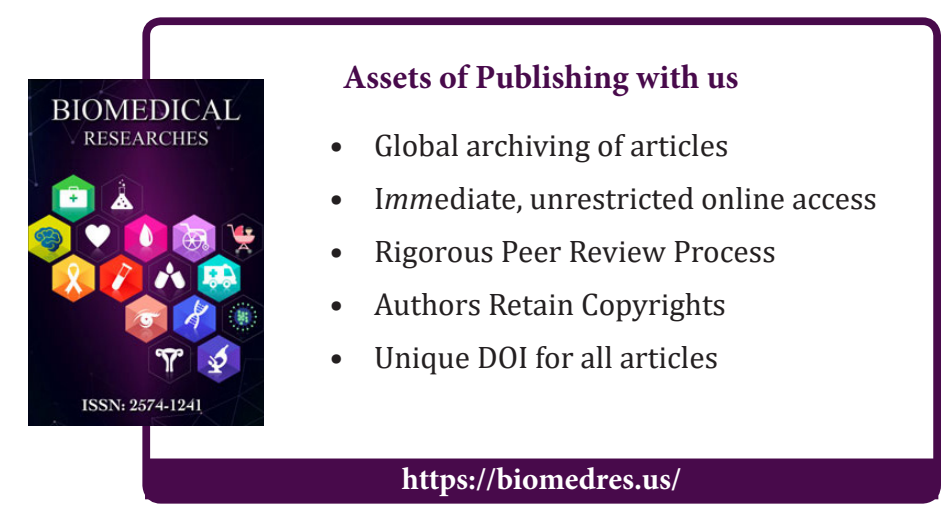

